\title{
Shock-driven gas curtain: fractal dimension evolution in transition to turbulence
}

\author{
Peter Vorobieff $^{\mathrm{a}, *}$, Paul M. Rightley ${ }^{\mathrm{b}}$, Robert F. Benjamin ${ }^{\mathrm{b}}$ \\ ${ }^{\text {a } C N L S / D X-3 / M S T-10, ~ M S ~ B-258, ~ L o s ~ A l a m o s ~ N a t i o n a l ~ L a b o r a t o r y, ~ L o s ~ A l a m o s, ~ N M ~ 87545, ~ U S A ~}$ \\ ${ }^{\mathrm{b}}$ DX-3, MS P-940, Los Alamos National Laboratory, Los Alamos, NM 87545, USA
}

\begin{abstract}
We present estimates of the Hausdorff fractal dimension of a planar section of the interfaces on the sides of a thin curtain of heavy gas $\left(\mathrm{SF}_{6}\right)$ embedded in air and accelerated with a planar shock wave at Mach 1.2. As the Richtmyer-Meshkov instability develops, eventually leading to a transition to turbulence in the curtain, the fractal dimension of the interfaces increases from an initial value not exceeding 1.10 to a maximum value $1.36 \pm 0.07$. This value is close to that experimentally measured for fully developed turbulence. The growth of the fractal dimension is closely related to mixing in the flow. It represents an important and previously unmeasured property in the transition to turbulence due to Richtmyer-Meshkov instability. (C1999 Elsevier Science B.V. All rights reserved.
\end{abstract}

Keywords: Fractals; Turbulence; Transition; Richtmyer-Meshkov instability; Experiment

\section{Introduction}

During transition to turbulence, an initially deterministic flow evolves to a state where individual flow features (e.g., interface shape) are unpredictable at least at some scales. However, the final, turbulent state of the flow can be characterized statistically. Some evidence suggests that the transition state may have predictable features as well. Despite the importance of transition to turbulence to a vast variety of hydrodynamical problems, a comprehensive theory of this phenomenon is yet to be developed, and even the phenomenological knowledge about it remains sparse.

\footnotetext{
* Corresponding author. New address after 16 August 1999: Department of Mechanical Engineering, University of New Mexico, Albuquerque, NM 87131, USA. Tel.: +1-505-277-2761, fax: +1-505-277-1571

E-mail address: kalmoth@rl66.com (P. Vorobieff)
}

Statistical theories typically assume homogeneity and isotropy and have limited applications even in the case of fully-developed turbulence. These theories do not offer a framework suitable for the understanding of the transition processes, which are significantly inhomogeneous, anisotropic and dominated by coherent spatial structures.

The specific problem of transition to turbulence induced by Richtmyer-Meshkov (RM) instability may serve as a useful test case for the more general studies. Several factors make RM particularly attractive as such a test case. This instability develops on an interface between a heavy and a light fluid subjected to an impulsive (shock) acceleration. It is analogous to the better known gravity-driven Rayleigh-Taylor (RT) instability. The most important difference between RM and RT is that the RM-unstable interface 'coasts' after the initial acceleration, whereas the growth of 
an RT-unstable interface is continuously driven by gravity. This leads to smaller growth rates for RM instability, simplifying the investigation of the transition region.

Experimental studies of RM instability typically provide sequences of flow visualization images. The experiments can be divided into two groups. The first group includes single-interface studies, starting with the original work by Meshkov [1]. More recently single-interface research was undertaken by Benjamin [2], Brouillette and Sturtevant [3], Dimonte et al. [4] and Velikovich and Dimonte [5]. Works in the second group, gas-curtain studies, deal with two nearby density interfaces resulting in a curtain of heavy gas (usually sulfur hexafluoride mixed with gaseous or aerosol tracer) embedded in a lighter gas (air). Investigations of the flow in a shock-accelerated gas curtain have been carried out by Jacobs et al. [6,7], Budzinski et al. [8], Rightley et al. [9,10] and Vorobieff et al. [11].

The state of the art in gas-curtain experimentation makes it possible to provide image sequences showing the initial condition of the curtain prior to shock impact and its subsequent evolution after the shock interaction, up to 30 images during a $800 \mu \mathrm{s}$ time. Thereby one can track the evolution of the curtain through three stages: first, the initial linear growth of the instability; second, the vortex-dominated nonlinear deterministic growth; third, the growth of disordered features and the eventual transition to turbulence.

What makes these experiments particularly valuable for transition studies is knowledge of the deterministic part of the flow. It is largely prescribed by the initial geometry of the system, because the mechanism of the initial vorticity production is baroclinic. As the shock passes through the density interface, vorticity is produced wherever the shock is not parallel to the interface due to the cross-product of the gradients of pressure $p$ and density $\rho$ in the vorticity equation. Knowledge of the initial conditions and the shock properties suffices to produce realistic estimates of the vorticity field that forms shortly after the shock interaction. The subsequent vortex dynamics can be simulated with a relatively simple 'vortex blob' model [9]. Thus, the emergence and evolution of the disordered features that leads to turbulence can be isolated and quantified in greater detail. The capability of a numerical simulation to predict complex flows can be discerned by the accuracy of code results on a range of scales at interfaces. A simple, quantitative method to compare experimental results with code predictions is fractal dimension analysis.

The measure of fractality of a turbulent flow, e.g., the fractal dimension of the interface between mixing gases, is the feature associated with small-scale disorder in turbulence [12]. It is particularly important for turbulent flows with mixing [13]. Fractal analysis in turbulence has a history practically as long as the history of fractal analysis itself. The multiscale nature of turbulence and apparent geometric complexity of turbulent flows attracted the attention of Mandelbrot [12], who suggested that the geometric structure of turbulence is fractal. Subsequent studies [14-18] showed that it is indeed possible to quantify the geometry of turbulent flows with power-law statistics in terms of a constant fractal dimension over a considerable range of scales. It is also of interest that in Mandelbrot's book [12], the opening of the chapter on turbulence (chapter 10 in the 1983 edition) mentions that transition to turbulence is likely to have 'fractal aspects of great importance, but they have not been clarified enough to be discussed here'. Since 1983, when the book was written, research on the fractal properties of fully developed turbulent flows has made significant advances, but the issue of the fractal features during the transition to turbulence remains largely unexplored.

The present work provides measurements of the evolution of the fractal dimension of a shock-accelerated gas curtain as the flow in it undergoes the transition to turbulence. We link these measurements with our previous studies investigating this transition in terms of enhanced mixing (as revealed by histogram analysis [10]), formation of multiple scales (wavelet analysis [10]), and in terms of the emergence of power-law behavior of the density structure function [11].

The gas-curtain experiments provide a clean and convenient test model for such transition studies. The mixing layer is impulsively accelerated. Its growth rate is lower than that for the case of constant acceleration. The flow is spatially inhomogeneous, anisotropic and initially compressible, yet the large-scale coherent 


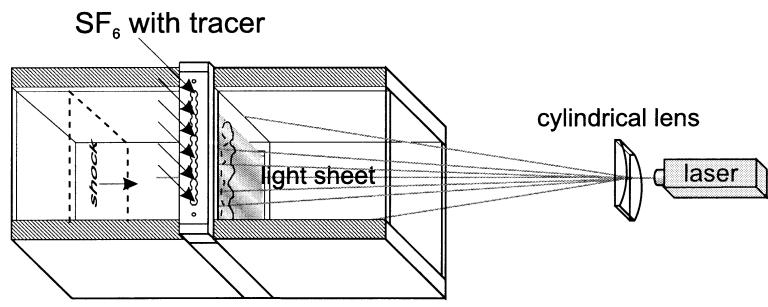

Fig. 1. Schematic of the test part of the driven section of the shock tube (top view).

structures driving the flow are known. The following sections provide the description of the experimental apparatus employed in our study and the discussion of our observations and data interpretation with fractal dimension analysis.

\section{Experimental setup and data acquisition}

The datasets analyzed in this paper were acquired in two series of shock-tube experiments. In both series, we employed the same Mach 1.2 shock tube with a $75 \mathrm{~mm}$ square cross-section. The mechanics of producing a planar shock are straightforward. The tube consists of two sections separated by a plastic diaphragm. One of the sections (driver) is pressurized. When the diaphragm is ruptured by a solenoid-driven puncturer, the shock propagates through the second (driven) section of the shock tube. A part of the driven section is equipped with view windows and a nozzle arrangement that produces the gas curtain (see Fig. 1). The shaped nozzle at the top imposes an initially varicose perturbation profile (wavelength $\lambda=6 \mathrm{~mm}$ ) upon the gas injected downward into the shock tube and removed through an exhaust plenum at the bottom. The velocity of the vertical flow in the curtain is of the order of $\mathrm{cm} / \mathrm{s}$ - negligibly small when compared with the shock-induced velocity $(100 \mathrm{~m} / \mathrm{s})$. Small random perturbations in the vertical flow lead to additional variations in the initial conditions, resulting in emergence of a variety of flow morphologies in the subsequent shock-accelerated flow [9].

The flow is illuminated with a laser sheet produced either with a continuous wave $(\mathrm{CW}) 1 \mathrm{~W}$ argon laser (constant illumination) or with a pulsed-laser strobe.
In the case of pulsed (strobed) lighting, images are acquired with a $512 \times 512$ Photometrics CCD camera. The maximum pulsing frequency of $5 \mathrm{kHz}$ limits the number of images acquired during one event to four (it takes about $1 \mathrm{~ms}$ for the shock-accelerated gas curtain to traverse the extent of the test section). The argon laser is used with a Hadland Photonics SVR camera which provides a better effective temporal resolution if the built-in gated intensifier is operated as a shutter - up to nine images per event. In both cases, it is possible to store multiple images (including that of the initial conditions) on one CCD because the mean flow carries the gas curtain downstream at $100 \mathrm{~m} / \mathrm{s}$, and with proper exposure timing multiple exposures can exist on the CCD without overlapping.

The curtain material, sulfur hexafluoride (five times the density of air), is mixed with a small volume fraction of a tracer (glycol fog) to facilitate flow visualization. A detailed description of the setup, including the analysis of the flow tracking fidelity of glycol fog, is provided by Rightley et al. $[9,10]$.

\section{Observations and analysis}

Fig. 2 shows two of the image sequences acquired in gas curtain experiments and analyzed in our previous works. The leftmost image in each sequence, denoted by 'IC' shows the initial conditions, and the labels of the subsequent images show corresponding times (in microseconds) since the shock passed the curtain. Black in the images corresponds to unmixed curtain material ( $\mathrm{SF}_{6}$ with glycol droplets), white to air.

The upper image sequence (Fig. 2(a)) shows the 'sinuous' [9] flow morphology evolution pattern developing from a regular, varicose initial condition with a moderate amplitude of perturbation $(\sim 2 \mathrm{~mm})$. The initial production of vorticity results in a row of vortices of alternating sign, entraining the curtain material to form 'mushroom' shapes facing alternatingly upstream and downstream. In all the images including the late-time ones, the flow appears vortex-driven and deterministic on the large scale, with a few disordered small-scale structures emerging at late times (the last two exposures). 

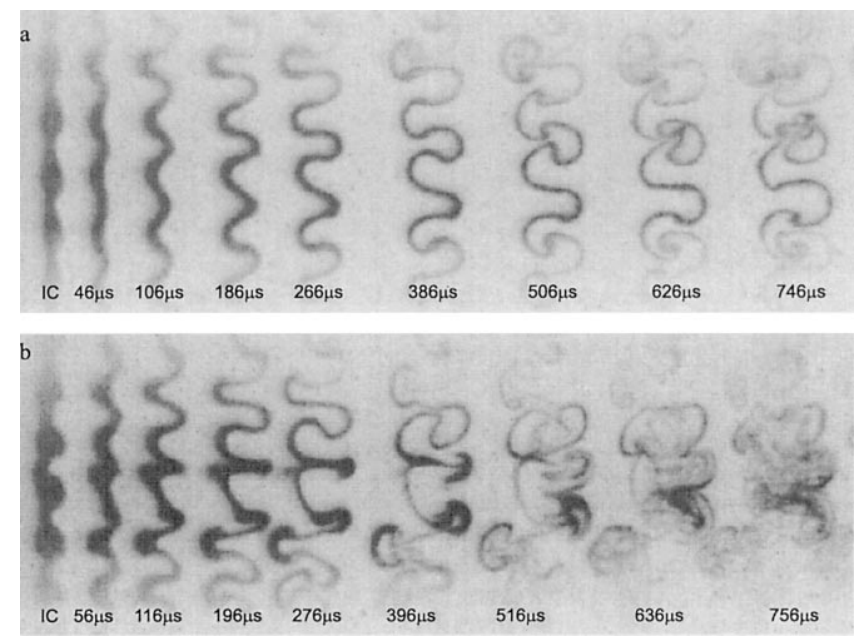

Fig. 2. Gas curtain flow morphologies: (a) sinuous, and (b) irregular. Labels denote exposure timings in microseconds after shock interaction. Duration of each exposure is $2 \mu \mathrm{s}$.

The lower image sequence, Fig. 2(b), is an example of an irregular flow morphology evolving from strongly perturbed (amplitude $\sim 3 \mathrm{~mm}$ ) initial conditions. In this case, the apparent growth of disordered features is significantly accelerated. In both cases, the deterministic large-scale flow is predominantly 2D, while the growth of the disordered small-scale features appears fully 3D. The 3D effects are more apparent in the irregular morphology cases. The results of the quantitative analysis of these and other sequences have been presented in our previous works $[10,11]$. The main differences between a regular and disordered evolution pattern are described below.

First, histogram analysis [10] shows that for the regular morphologies, including the case shown in Fig. 2(a), the mixing of the curtain material with air is gradual and occurs at approximately the same rate throughout the recorded duration of the experiment (about $800 \mu \mathrm{s}$ ). Irregular morphologies, on the other hand, are often characterized by acceleration of mixing. For the experiment presented in Fig. 2(b), such an acceleration ('mixing transition' [10]) occurs between the fifth $(400 \mu \mathrm{s})$ and sixth $(520 \mu \mathrm{s})$ dynamic exposure, when approximately $40 \%$ of the previously unmixed curtain material abruptly mixes with air.
Second, results of wavelet analysis [10] are qualitatively similar for the regular and irregular morphologies; however, the wavelet signature of the coherent structures (mushroom shapes) erodes faster in the irregular morphology case.

Third, the second-order structure function of density (density-density correlation function) develops a power-law behavior with scaling close to $2 / 3$ for irregular morphologies [11]. A similar scaling is likely to evolve for regular morphologies, although the limited time duration of the experiment is insufficient to see the completion of this process - again, regular morphologies evolve towards turbulence at a slower rate than irregular morphologies.

What are the fractal dimension characteristics associated with this evolution and how do they vary between regular and disordered flow patterns? Before answering this question, let us describe the method we employ to estimate the fractal dimension of the gas curtain interfaces with surrounding air. We use a straightforward implementation of a box-counting algorithm, the description of which can be found, for instance, in the review by Theiler [19], whose arguments we follow below to introduce the fractal dimension.

Let $\mathcal{A}$ be the fractal we analyze, and $\mathcal{C}(r, \mathcal{A})=$ $\left\{B_{1}, \ldots, B_{K}\right\}$ be a finite covering of $\mathcal{A}$ with 
simply-connected $B_{i}$ such that the $\delta_{i}$, the diameter of each $B_{i}$, is less than $r$. Introduce a coarse-grained measure $\Gamma$

$\Gamma(\mathcal{A}, D, r)=\min _{\mathcal{C}(r, \mathcal{A})} \sum_{i} \delta_{i}^{D}$

where $D$ is the dimension of the measure and min represents the minimum over all coverings for which $\delta_{i}<r$. For most values of $D$, this measure is degenerate (zero or infinity) in the limit $r \rightarrow 0$. For a 1D object (a curve) and $D>1, \Gamma \rightarrow 0$. For a finite 2D area and $D<2, \Gamma \rightarrow \infty$. In general, one can show the behavior of $\Gamma(\mathcal{A}, D, r)$ as $r \rightarrow 0$ to satisfy the equation

$\Gamma(\mathcal{A}, D)=\lim _{r \rightarrow 0} \Gamma(\mathcal{A}, D, r)= \begin{cases}\infty, & D<D_{\mathrm{H}} \\ 0, & D>D_{\mathrm{H}},\end{cases}$

where the transition point $D_{\mathrm{H}}$ for which a nondegenerate limit exists is the Hausdorff dimension of the fractal $\mathcal{A}$.

In reality, the requirement to take the minimum over all coverings in Eq. (1) is usually relaxed, and the covering selected to estimate the upper bound on the Hausdorff dimension is just a fixed-sized grid. If this is the case for an $r$-sized grid, Eq. (1) transforms into

$\Gamma(\mathcal{A}, D, r)=\sum_{i} \delta_{i}^{D}=\sum_{i} r^{D}=n(r) r^{D}$,

where $n(r)$ is the number of nonempty grid boxes. The non-degenerate $\Gamma\left(\mathcal{A}, D_{\mathrm{H}}\right)$ would then mean that

$\lim _{r \rightarrow 0} \Gamma\left(\mathcal{A}, D_{\mathrm{H}}, r\right)=\lim _{r \rightarrow 0} n(r) r^{D_{\mathrm{H}}}$

is finite. To make this possible, it must hold that

$D_{\mathrm{H}}=\lim _{r \rightarrow 0} \frac{\log [1 / n(r)]}{\log r}$.

The actual range of scales over which this fractal behavior is observed is limited by the inner and outer cutoff scales. The $r \rightarrow 0$ limit is not realized in estimating the fractal dimension of physical objects [19]. For turbulent non-reacting fluid flows, Mandelbrot suggested the Kolmogorov scale as the value of the inner cutoff and the characteristic size of the apparatus as the outer cutoff [12].
Now let the time-dependent curve $\mathcal{A}(t)$ be the boundary of the level set of constant intensity $h>h_{b}$ corresponding to a level set of $\mathrm{SF}_{6}$ concentration in one of the gas curtain image sequences. We select the central part of the curtain, where the intensity falloff from the illumination nonuniformity is negligible. The box size $(30 \mathrm{~mm})$, about half the width of the test section of the shock tube, is the outer cutoff scale. Because the image has only a finite pixel resolution, the pixel size has to serve as the inner cutoff. Within this scale range, we estimate $D_{\mathrm{H}}$ numerically following Eq. (5) for each image in the sequence by plotting $\log [1 / n(r)]$ versus $\log r$ and measuring the slope and the error of the linear fit. Each of the boundaries includes the upstream and downstream interface. To check for the possible influence of rasterization/box size on the results, we also calculated $D_{\mathrm{H}}$ using only the data within a smaller scale range - from two pixel sizes to half the box size. This would amount to discarding the first and the last data points in the $\log [1 / n(r)]-\log r$ plot. The change in $D_{\mathrm{H}}$ estimates that such a truncation introduced was on the order of $1 \%$.

We present data for boundaries of four level sets. The first level set represents the area with the concentration of curtain material $\left(\mathrm{SF}_{6}\right.$ with fog) $7 \%$ or greater - the minimal concentration we can accurately resolve. Similarly, the subsequent sets correspond to 14.5, 22 and 29\% concentrations. For both image sequences, the fractal dimension of the initial conditions in most level sets does not exceed 1.10. It is somewhat greater than unity because the interface is diffuse. Level sets of the last dynamic exposure from the image sequence in Fig. 2(b) are presented in Fig. 3.

Late-time results for the 22 and $29 \%$ concentrations may underestimate $D_{\mathrm{H}}$ because of limited spatial resolution - with a sufficient fraction of the curtain material mixed with air, the 'islands' of higher concentration become small and may not provide enough information for a good estimate of the fractal dimension. Hence, we base most of our further analysis on the first two level sets.

Fig. 4 presents the estimated box-counting dimensions and linear fit errors for the image sequences in Fig. 2. Results of the analysis of several other simi- 

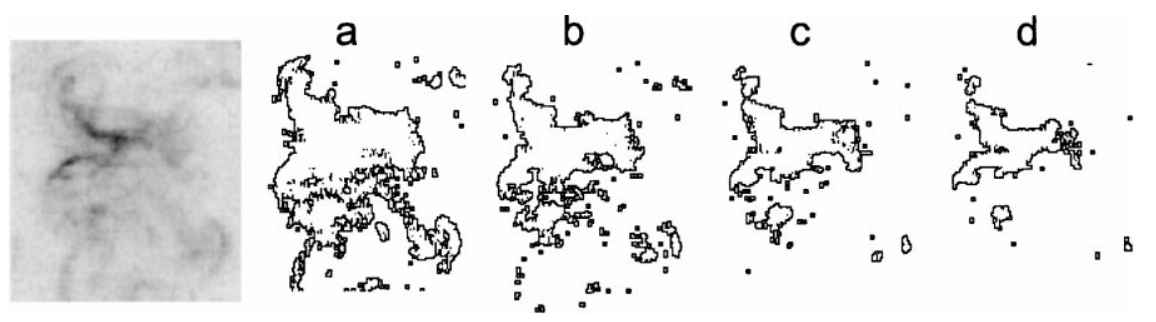

Fig. 3. Level sets for the last dynamic exposure, irregular morphology (Fig. 2(b)): (a) boundary of level set with curtain material concentration $7 \%$ or greater, (b) same for $14.5 \%$, (c) $22 \%$, and (d) $29 \%$. The corresponding fractal dimension $D_{\mathrm{H}}$ estimates are: (a) $1.37 \pm 0.02$, (b) $1.31 \pm 0.02$, (c) $1.26 \pm 0.02$, and (d) $1.21 \pm 0.03$.
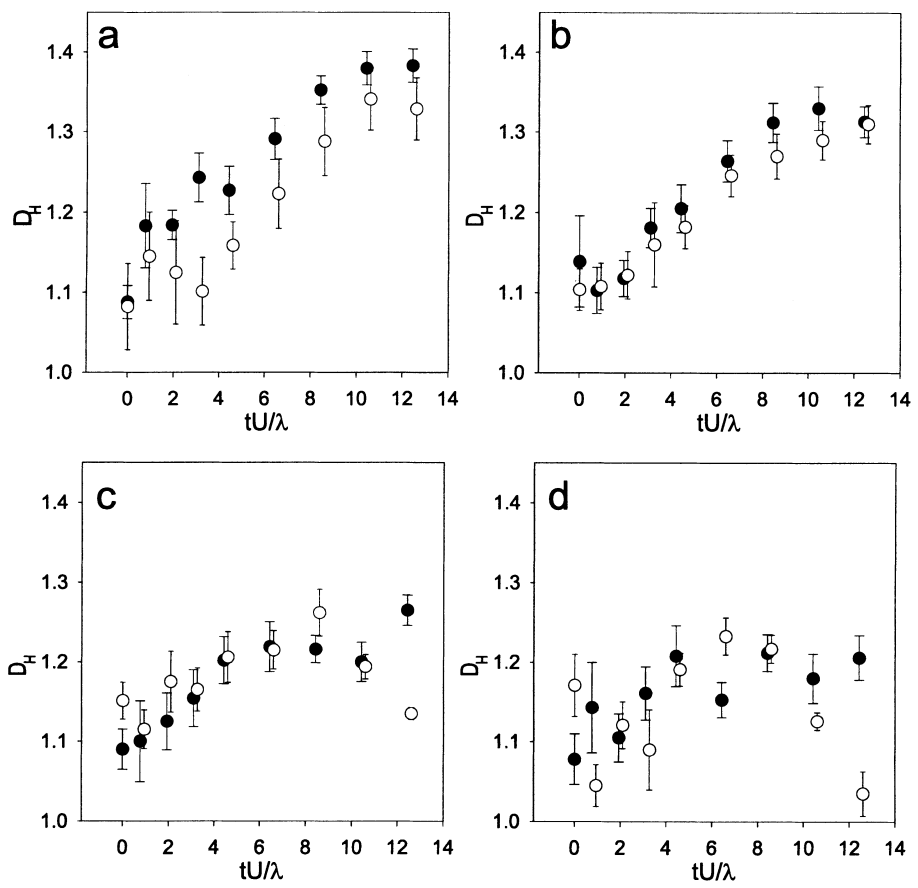

Fig. 4. Evolution of fractal dimension estimate $D_{\mathrm{H}}$. ०: sinuous morphology (Fig. 2(a)), O: irregular morphology (Fig. 2(b)), (a) boundary of level set with curtain material concentration $7 \%$ or greater, (b) same for $14.5 \%$, (c) $22 \%$, and (d) $29 \%$.

lar regular and disordered evolution patterns we processed, are very close to the ones shown here. The time in the plot is nondimensionalized with a combination of piston velocity $U=100 \mathrm{~m} / \mathrm{s}$ and the fundamental wavelength $\lambda=6 \mathrm{~mm}$.

For lower concentrations (Figs. 4(a,b)), the results can be summarized as follows. The irregular morphology is characterized by the fractal dimension apparently approaching values $1.37 \pm 0.02$ for $7 \%$ concentration, Fig. 4(a), and $1.31 \pm 0.02$ for $14.5 \%$ concentration, Fig. 4(b). Other irregular morphology data sets show a similar behavior, with the late-time value of $D_{\mathrm{H}}$ averaged for four image sequences being $1.36 \pm 0.07$ at $7 \%$ concentration. It is noteworthy that the change in the behavior of $D_{\mathrm{H}}$ (initially near-linear growth slows down) occurs between the fifth and sixth dynamic exposures - the same time interval when, according to histogram analysis data, $40 \%$ of the previously unmixed curtain material mixes with air. Our previous study [10] suggests that this accel- 


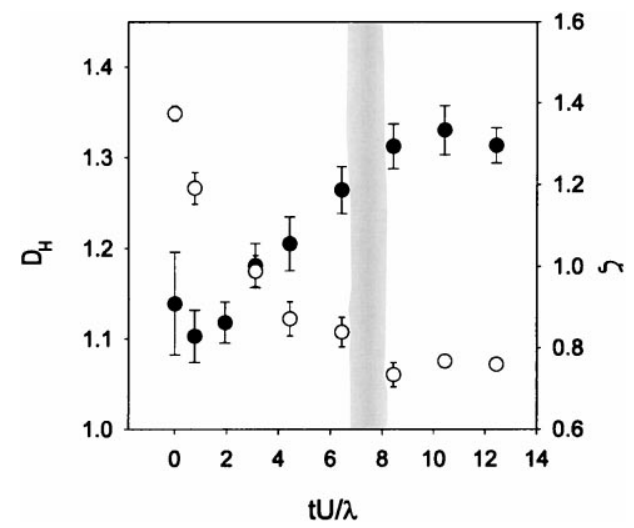

Fig. 5. Irregular morphology: time history of $D_{\mathrm{H}}$ for the $14.5 \%$ level set boundary (left vertical axis, - ) and of $\zeta$, the exponent of the power-law fit to the second-order structure function of intensity $I_{2}(l)$ from Eq. (6) (right vertical axis, o). Gray area indicates the 'mixing transition' time interval according to histogram data.

eration of mixing is a sign of the 'mixing transition' $[20,21]$. To further illustrate the relationship between the 'mixing transition' and the evolution of other curtain properties for the irregular morphology, in Fig. 5 we compare the fractal dimension of the $14.5 \%$ level set boundary shown in Fig. 4(b) with the exponent $\zeta$ of a power-law fit to the second-order structure function of intensity

$I_{2}(l)=\left\langle[I(x+l)-I(x)]^{2}\right\rangle \sim l^{\zeta}$,

where $\langle\cdot\rangle$ denotes spatial averaging in the image plane for all points $\boldsymbol{x}=(x, y)$ and all 2D radius-vectors $\boldsymbol{l}, \| \boldsymbol{l} \mid<1.5 \lambda . I_{2}(l)$ can be considered as a real-space analog of density spectrum or as the density-density correlation [11]. The rates of change of both the exponent of the power-law fit and the fractal dimension of the level set boundary decrease drastically after the 'mixing transition' (gray vertical area in the plot) occurs. After this transition, the slope of the power-law fit approaches $2 / 3$ - the value associated with fully developed turbulence [11].

Before the 'mixing transition' occurs during the evolution of the irregular flow pattern, the behavior of the fractal dimension for the sinuous morphology is similar to that for the irregular morphology, but with a time delay of the order of 1-2 units of dimensionless time $(60-120 \mu \mathrm{s})$. No evidence of 'mixing transition' is present in the sinuous morphology data, and there is no apparent slow-down in the rate of change of the fractal dimension. The last two data points in Fig. 4 for the sinuous morphology may show the beginning of slowing down, but the time interval of our observations is insufficient to make a conclusion. This delay is consistent with the notion that sinuous morphology evolves towards turbulence more slowly, in agreement with the results acquired with different methods of analysis [9-11]. Shock interaction with regular initial conditions produces relatively less vorticity, leading to slower evolution.

The behavior of level sets for higher concentrations (Figs. 4(c,d)) at early times is similar to the growth described above for lower-concentration sets. At late times, however, the fractal dimension of higher-concentration sets decreases. Some of this decrease is caused by mixing of curtain material with air, some may also be an effect of the loss of resolution. Here, we must note an interesting parallel with the work of Flohr and Olivari [18]. Their study visualized planar sections of a turbulent jet with a passive scalar (smoke) advected by the jet flow. The fractal dimension of the level sets of scalar concentration estimated by Flohr and Olivari [18] goes down with concentration, similar to our late-time results.

For lower concentrations, Flohr and Olivari reported a fractal dimension $1.30 \pm 0.05$, which is reasonably close to our late-time result for irregular morphology $(1.36 \pm 0.07)$. Other experimental results for fractal dimension of 2D sections of scalar level-set interfaces in fully developed turbulent jet and mixing-layer flows agree with ours even better: $1.36 \pm 0.05$ [15], 1.32-1.4 [14]. This agreement is important not only because our flow is transitional, but also because we work with density level sets in a spatially inhomogeneous flow rather just with level sets of a passively advected scalar. We must also note that the Reynolds numbers of the flows studied by Flohr and Olivari (1000-3000) and Prasad and Sreenivasan (up to 15,000) are lower than that of our flow (conservative lower-bound estimate 20,000 [10]). This may provide additional confirmation for the observation of Prasad and Sreenivasan [15] that the dimension of the interface (1.36) is inde- 
pendent of the Reynolds number for sufficiently high values of the latter.

\section{Conclusions}

In our study of RM instability of a shock-accelerated gas curtain, we investigate the time evolution of the Hausdorff dimension $D_{\mathrm{H}}$ characterizing 2D lines bounding the level sets of the concentration of the curtain material, heavy gas $\left(\mathrm{SF}_{6}\right)$ mixed with tracer (glycol fog). We observe that the time evolution of $D_{\mathrm{H}}$ correlates well with other diagnostics of the mixing transition in gas curtain flows. $D_{\mathrm{H}}$ initially has values less than 1.10 for both the irregular and regular cases. For irregular flow morphologies (multiple, random modes initially), earlier works found that visual, correlation-function and histogram analyses all show the 'mixing transition' occurring between 400 and $520 \mu$ s after shock-acceleration of the gas curtain, and we now find that after the transition $D_{\mathrm{H}}$ approaches a value between 1.3 and 1.4 , close to that reported in a number of studies for the fractal dimension of interfaces in fully-developed turbulent flows. At the same time, $D_{\mathrm{H}}<1.3$ and is still increasing for regular morphologies, which is consistent with visual, correlation-function and histogram evidence that mixing is not yet extensive in this case. We expect the 'mixing transition' to occur relatively late for regular morphologies in comparison with irregular morphologies because baroclinic vorticity production is more disordered for irregular morphologies, and we observe that the growth of $D_{\mathrm{H}}$ supports this expectation. Fractal dimension analysis agrees with three other analysis techniques in identifying the 'mixing transition'. We suggest that the 'mixing transition' is an indicator of incipient turbulence both in terms of the spectra of density fluctuations (correlation function) and the geometry of interfaces (fractal dimension).

\section{Acknowledgements}

We thank Frank Kosel and Hadland Photonics, for the use of the SVR camera. We are grateful to Shiyi Chen, Richard N. Silver, William J. Rider, James R. Kamm and Charles W. Cranfill for their helpful suggestions regarding this article.

\section{References}

[1] E.E. Meshkov, Izv. Akad. Nauk SSSR - Mekh. Zhidk. Gaza 4(5) (1969) 101.

[2] R.F. Benjamin, in: R. Dautray (Ed.), Proc. 3rd Int. Workshop on the Physics of Compressible Turbulent Mixing, Abbey of Royaumont, France, 17-19 June 1991, CEA, Paris, 1991, p. 325.

[3] M. Brouillete, B. Sturtevant, Phys. Fluids A 5 (1993) 916.

[4] G. Dimonte, C.E. Frerking, M. Schneider, Phys. Rev. Lett. 74 (1995) 4855.

[5] A.L. Velikovich, G. Dimonte, Phys. Rev. Lett. 76 (1996) 3112.

[6] J.W. Jacobs, D.L. Klein, D.G. Jenkins, R.F. Benjamin, Phys. Rev. Lett. 70 (1993) 583.

[7] J.W. Jacobs, D.G. Jenkins, D.L. Klein, R.F. Benjamin, J. Fluid Mech. 295 (1995) 23.

[8] J.M. Budzinski, R.F. Benjamin, J.W. Jacobs, Phys. Fluids 6 (1994) 3510

[9] P.M. Rightley, P. Vorobieff, R.F. Benjamin, Phys. Fluids 9 (1997) 1770

[10] P.M. Rightley, P. Vorobieff, R.F. Benjamin, R. Martin, Phys. Fluids 11 (1999) 186.

[11] P. Vorobieff, P.M. Rightley, R.F. Benjamin, Phys. Rev. Lett. 81 (1998) 2240.

[12] B.B. Mandelbrot, The Fractal Geometry of Nature, Freeman, New York, 1983.

[13] H.J. Catrakis, P.E. Dimotakis, Phys. Rev. Lett. 77 (1996) 3795.

[14] K.R. Sreenivasan, C. Meneveau, J. Fluid Mech. 173 (1986) 357.

[15] R.R. Prasad, K.P. Sreenivasan, Phys. Fluids A 2 (1990) 792.

[16] K.R. Sreenivasan, Annu. Rev. Fluid Mech. 23 (1991) 539.

[17] P. Constantin, I. Procaccia, K.R. Sreenivasan, Phys. Rev. Lett. 67 (1991) 1739.

[18] P. Flohr, D. Olivari, Physica D 76 (1994) 278.

[19] J. Theiler, J. Opt. Soc. Am. A 7 (1990) 1055.

[20] J.C. Lasheras, J.S. Cho, T. Maxworthy, J. Fluid Mech. 172 (1986) 231.

[21] L.P. Bernal, A. Roshko, J. Fluid Mech. 170 (1986) 499. 